\title{
Communicating ALMA with the Public in Japan
}

\author{
M. Hiramatsu \\ National Astronomical Observatory of Japan, 2-21-1, Osawa, Mitaka, Tokyo, 181-8588 Japan \\ email: hiramatsu.masaaki@nao.ac.jp
}

\begin{abstract}
I present the strategy and activities of the public outreach and communication of ALMA in Japan. Since most of the public is not familiar with the radio astronomy, we present the human side of ALMA to attract the public interest, as well as by showing the science results. To evoke the public interest on the radio astronomy, it is also effective to show the radio astronomy research topics on the planets, the Sun, and bright stars so that they can connect the daily night sky with the radio universe explored by ALMA.
\end{abstract}

\section{ALMA Outreach Strategy in Japan}

ALMA is the powerful radio telescope constructed in the Chilean Andes with the international partnership of Europe, North America and East Asia in cooperation with the Republic of Chile. Public outreach activities become more and more important with the progress of the construction and start of science operation in September 2011. Here I describe the outreach and public communication of ALMA in Japan.

The outreach of ALMA in Japan is difficult in the following points; Chile is distant from Japan, the public is not familiar with radio astronomy, and even less with radio interferometer. To overcome these difficulties, our strategy is not only to show the science results but also to "tell the stories" of the cool universe, ALMA staff members, and high technology employed by ALMA. Showing the human side of ALMA, for example "What do astronomers want to know with ALMA?", "How is the life in the ALMA observatory?", "How did the engineers build the great telescope with cutting-edge technologies?" initiates the interest of ALMA among those who are not interested in astronomy or science.

\section{ALMA Outreach Activity in Japan}

Along with the public lectures and science cafes, we make use of the online tools; web site, twitter, facebook, and mail magazine. To showing the "human face", we have the photo reports of the construction progress, the serial online articles written by the NAOJ ALMA staff members to introduce their daily life and work in Chile, and short movies of the antenna construction factories with the interviews of the craftsmen.

With twitter, we tweet the news updates of ALMA construction and scientific results, announcements of public talk, as well as notices of celestial events such as eclipses and planet conjunctions. The number of retweets and favorates is the largest for the last topic, which shows many people are interested in the visible sky and want to share those information. They look unrelated to ALMA, but the planets and the Sun are also ALMA's important targets. Together with the guidance of celestial events, we tweet the research topics on the Sun, planets, exoplanets, and so on, which would be explored by ALMA. Those tweets obtain larger number of "retweets" and "favorates", and this helps the public connect the daily night sky with the radio universe. 